\title{
Editorial Dossier
}

\section{Conflictos y resistencias al neoliberalismo en América Latina}

Verónica Giordanoํㅜ ${ }^{1}$ Miguel Leone ${ }^{2}$

En la historia de las sociedades latinoamericanas siguen vigentes los embates del colonialismo, el patriarcado y la violencia de los despojos. Por eso, también siguen activas y pujantes las luchas de los pueblos y las resistencias colectivas construidas e inscriptas en tiempos de larga duración.

En este marco, desde la década del noventa del siglo pasado, el avance de las políticas neoliberales marcó una nueva coyuntura histórica. El Estado de compromiso social quedó finalmente sepultado y el capital entró de lleno en la fase de financiarización del capital. El achicamiento del Estado y la acumulación ampliada del capital - como nunca antes había sido visto en la historia del capitalismo-, se impusieron a escala global.

En América Latina, la creación de instrumentos financieros con exorbitantes ganancias privadas financiadas por el erario público (en buena medida, a través de la generación de deuda externa), la privatización de empresas -incluso aquellas de carácter estratégico para el desarrollo de las economías nacionales-, y el desarrollo de nuevas formas de acumulación originaria, esta vez de tipo neoextractivista, son algunos de los rasgos centrales que ha adquirido el neoliberalismo en el plano económico.

1 Doctora en Ciencias Sociales. Investigadora del Consejo Nacional de Investigaciones Científicas y Técnicas (CONICET- Argentina). Instituto de Estudios de América Latina y el Caribe, Universidad de Buenos Aires. Buenos Aires, Argentina. E-mail: veronicaxgiordano@gmail.com

2 Doctor en Ciencias Sociales. Becario Posdoctoral de la Universidad de Buenos Aires. Instituto de Estudios de América Latina y el Caribe, Universidad de Buenos Aires. Buenos Aires, Argentina. E-mail: miguelleone@hotmail.com 
Si como postuló Marx el capitalismo es un modo de producción revolucionario, puesto que constantemente re-evoluciona los medios de producción, se puede afirmar provocativamente que han sido "revolucionarias" las veloces transformaciones de las tecnologías de cultivo producidas de la mano de la transgenia desde los años ochenta y hasta el presente. Y con ello han sido realmente profundos los cambios en el espacio rural. Pequeños/as productores/as agropecuarios/as ${ }^{3}$, indígenas, campesinos/as se han visto forzados a abandonar sus tierras y/o resistir al despojo en condiciones de extrema desigualdad.

En este marco, América Latina se convirtió en escenario de nuevos conflictos y laboratorio de inéditas formas de resistencia, tanto rurales como urbanas, capaces de replantear el juego de las marcaciones (y des-marcaciones) de clase, de género, y de las identidades étnicas y los procesos de racialización.

En efecto, desde fines del siglo XX, han surgido importantes movimientos sociales en América Latina. Suele indicarse como punto crucial de inicio de esta tendencia el surgimiento del movimiento zapatista en México en 1994. No obstante, es evidente que este punto recoge y condensa experiencias previas como el Movimento Sem Terra en Brasil o La Vía Campesina nacida en Nicaragua en 1992. Ya en el siglo XXI, también es posible observar creativas y originales luchas populares, en donde sin duda destacan los movimientos feministas, de mujeres y de género que están bregando por la ampliación de derechos y el fin de la violencia patriarcal en distintas dimensiones y aspectos, de los cuales destaca la interseccionalidad y transversalidad.

Este dossier propone, a través de sus variados artículos, analizar los conflictos y resistencias al neoliberalismo en el contexto espacio-temporal más amplio que describimos anteriormente. Así, las experiencias y situaciones que se analizan, los cuerpos y las subjetividades, aparecen ancladas en un tiempo y espacio particular, siempre engarzadas con estructuras más amplias. Las construcciones teóricas acerca del conflicto y el cambio son tan necesarias como la investigación histórica concreta, y particularmente cuando estas son elaboradas desde

3 La Revista Temas Sociológicos ha respetado en la edición de este artículo el uso de “/os/as" en lugar del masculino genérico privilegiado por la RAE para referirse a la clase que corresponde a todos los individuos de la especie, sin distinción de género. 
y para América Latina. Como sostiene Tilly (1991), es necesario un regreso a los núcleos matrices de las disciplinas "que supone, por un lado, ocuparse de identificar y conceptualizar procesos de cambio social en gran escala, y por el otro, de analizar los hechos en la menor escala posible" (p. 30).

Por otro lado (Giordano, 2011), hemos hecho un alegato a favor de la sociología histórica comparada, y hemos afirmado que una apelación a ella no equivale (o no debería hacerlo) a rechazar la existencia de hechos únicos e irrepetibles y la fertilidad de los estudios orientados a un solo caso. Antes bien, la comparación en sociología histórica debe buscar la comprensión de esos hechos únicos e irrepetibles dentro de modelos que van más allá de la singularidad. Sin duda, para ello es necesario contar con fuentes de información que permitan elaborar datos en ese nivel y escala de generalización.

El espíritu del dossier que aquí presentamos tiene la vocación de aportar al debate referente a los conflictos y resistencias al neoliberalismo de América Latina, entendiendo que el presente es histórico, es decir, es parte de una estructura temporal de más larga duración. Así mismo, también tiene la vocación de poner en circulación y diálogo un análisis de los fenómenos situados en tiempos y espacios singulares que puedan alimentar miradas de conjunto sólidamente informadas.

La noción de hegemonía resulta particularmente provechosa para la tarea. En efecto, toda construcción hegemónica es un espacio abierto en el ejercicio del poder, que se cifra en la lucha de clases, se juega en la materialidad de los cuerpos y se sitúa en territorios y espacios colectivos. La hegemonía es por definición, inestable, y para captar esa inestabilidad es preciso leerla en los diversos puntos críticos en que se dan las disputas. Para ello queremos, a continuación, brindar algunos puntos de anclaje de escala regional que permitan encuadrar los análisis que presenta este dossier en una trama temporal de más largo aliento.

Cuando Luíz Inácio Lula Da Silva asumió la presidencia de Brasil (2002) y Néstor Kirchner hizo lo propio en Argentina (2003), comenzó a cristalizarse una idea de integración regional, apoyada fuertemente en el presidente venezolano Hugo Chávez (en el cargo desde 1998). En 2004 fue lanzada la Alianza Bolivariana para los Pueblos de Nuestra 
América (ALBA), la cual funcionó como plataforma de contestación a la política de libre comercio impulsada por la Casa Blanca de Estados Unidos. Puede situarse en este punto de escala regional el acontecimiento crucial para comprender "el giro progresista" en varios países de la región.

Más tarde, la llegada de Evo Morales a la presidencia de Bolivia (2006) y Rafael Correa en Ecuador (2007) terminaron de delinear el perfil de lo que, con diferentes sentidos y énfasis, se afirmó con la categoría de Nuestra América. Los procesos constitucionales de esos dos países sellaron el reconocimiento de la plurinacionalidad como pauta de reparación histórica de unas sociedades fuertemente violentadas por el colonialismo. Por supuesto que los movimientos sociales de demanda de reconocimiento de la diversidad cultural tenían una historia de luchas previas e incluso exteriores o enfrentadas a las identidades político-partidarias que encabezaron las transformaciones constitucionales referidas. Sin embargo, queremos marcar el momento de sanción de las nuevas Constituciones como un hito de escala regional que permite mirar los procesos de aceleración de los conflictos y de cambios en una perspectiva regional (o de "unidad en la diversidad" para retomar una metáfora recurrente en las ciencias sociales latinoamericanas).

El proceso de integración regional dio un paso más a partir de la firma, en 2008, del acuerdo de creación de la Unión de Países Sudamericanos (UNASUR). Fue, en definitiva, un proceso que se plasmó en este tipo de acuerdos, pero se apoyó en alianzas transfronterizas. De estas alianzas participaron otros dos gobiernos del "ciclo progresista", el de Tabaré Vázquez en Uruguay (2005) y el de Fernando Lugo en Paraguay (2008).

Concomitantemente, la oposición a estas nuevas alianzas de poder y nueva expresión del orden social comenzó a aglutinarse en el polo derecho del arco político. La crisis del mensalão en Brasil ${ }^{4}$, la crisis del campo en Argentina y, más contundentemente, los golpes de estado en Honduras (2009) y en Paraguay (2012) fueron los primeros indicios

4 Escándalo de las mensualidades (en portugués, Escândalo do Mensalão) es el nombre dado a la crisis política sufrida por el gobierno brasileño en 2005 en relación con un caso de corrupción política en la Cámara de Diputados de Brasil. 
de la capacidad de acción de las derechas en América Latina. Si bien el carácter golpista de estas fuerzas tiene características diferentes a las desplegadas a partir de los golpes de los años setenta, ellas mantienen intacta su vocación destituyente y antidemocrática. A los casos-laboratorio de Honduras y Paraguay (dos casos que podrían considerarse "menores") les siguió el gigante del sur: Brasil en 2015 y más recientemente el caso de Bolivia en 2019, un caso emblemático por la transformación del orden desplegada y condensada en la nueva forma de Estado asumida en ese país.

La reacción de las derechas obedece a un aspecto clave: la igualdad. Las políticas orientadas al fortalecimiento de la inclusión social y la redistribución de la riqueza, la mitigación de la desigualdad social y, en algunos casos, la reestatización de empresas y recursos esenciales de nuestras sociedades llevadas adelante por los gobiernos denominados "progresistas" fueron el blanco de la reacción. El tenor de esta tensión operó como un fuerte límite para el cumplimiento de las consignas antineoliberales levantadas, con mayor o menor vehemencia, por esos gobiernos durante la primera década del siglo XXI.

En efecto, para entender el éxito de las fuerzas políticas neoliberales debe atenderse al poder de los grupos económicos altamente concentrados de la región. Estos grupos fueron aliados clave de sujetos políticos muchas veces aglutinados en nóveles partidos políticos o poderosos tanques de pensamiento, orientados por un objetivo primordial: destruir las conquistas sociales obtenidas en los primeros tres lustros del siglo XXI y profundizar la desigualdad social.

Con la asunción de Mauricio Macri como presidente de Argentina (en diciembre de 2015), Iván Duque en Colombia (en agosto de 2018) y Jair Bolsonaro en Brasil (enero de 2019) se puede decir que el "ciclo progresista" se cerró en América Latina, pero -más preocupantemente- dejó de manifiesto la capacidad de las derechas en la región para reconfigurar sus estrategias de poder y proyectarse hacia adelante.

Un dato llamativo de la reconversión estratégica es el acceso directo de las burguesías a las estructuras del Estado, dando lugar a lo que se ha dado a conocer como "gobierno de los empresarios" o "ceocracia", todas expresiones conocidas en la historia de América Latina desde los estudios de la oligarquía que mencionan la captura del Estado 
como un rasgo característico. También fue importante en este juego de reordenamientos, la mayor articulación con las ONG, fundaciones y think tanks de diverso tipo; o también -intentando "capturar" los espacios de legitimidad generados por los movimientos feministasel mayor protagonismo del perfil de las presidentas y vicepresidentas en las fórmulas de las fuerzas de las derechas. El caso de Jeanine Áñez en Bolivia es paradigmático, siendo esta mujer el rostro femenino de la derecha racista, que no dudó en torturar y asesinar a cholas, mineros y militantes del MAS, así como impulsar la conformación de grupos parapoliciales para ejecutar la violencia. Si el caso de Añez es paradigmático, no menos relevantes son los casos de María Gabriela Michetti, vicepresidenta del mandato de Macri en Argentina o Marta Lucía Ramírez, vicepresidenta del mandato de Duque en Colombia (Giordano y Rodríguez, en prensa).

Chile es un caso particularmente interesante en este escenario. Allí, la militarización de las policías y la policialización de las Fuerzas Armadas ha sido un recurso histórico para perseguir y criminalizar la protesta social. La violencia del régimen pinochetista se observa hoy por otras vías. Por ejemplo, en la militarización del territorio mapuche que, con mayor énfasis desde el año 2000, opera como una perversa forma de gestión de las demandas indígenas por parte de las derechas (Leone, 2018; en prensa).

La política de represión y muerte implementada por el gobierno de Sebastián Piñera contra la contundente movilización social de octubre de 2019 también requiere ser leída en esa trama histórica. El caso muestra a todas luces que la política de militarización de las derechas en Chile (y en América Latina) es un rasgo de continuidad respecto de las derechas golpistas de los años setenta, ahora vestidas con ropaje democrático.

En este contexto, vale la pena reponer una pregunta formulada en otro lado: ¿nuevas derechas respecto de qué pasado? (Giordano, 2014). No queremos desestimar las líneas de ruptura ni las diferencias sustantivas, solo queremos advertir que en las miradas de larga duración emergen continuidades que vale la pena atender para no teñir de absoluta novedad hechos que, inscriptos en una historia de más larga duración, pueden ofrecernos claves explicativas, e incluso lecciones, para el presente. Este es también el espíritu del dossier. 
Pero el giro a la derecha no es imbatible. En Argentina, Macri no tuvo éxito en su pretensión de renovar su mandato y perdió las elecciones frente al candidato del Frente de Todos, Alberto Fernández, quien triunfó nada menos que con la exmandataria Cristina Fernández de Kirchner en la vicepresidencia. También Chile resulta, nuevamente, un caso ilustrativo, aunque esta vez para dar cuenta de la capacidad política de las resistencias. Las masivas protestas sociales de octubre de 2019 derivaron (luego de violentos episodios que dejaron un saldo elevado de muertes y mutilaciones) en la reciente aprobación mediante plebiscito de una convocatoria a una Asamblea Constituyente para reemplazar la Constitución de 1980, pergeñada por el pinochetismo durante la dictadura. El hecho es alentador, aun cuando el gobierno de Piñera parece haber articulado un blindaje exitoso para limitar el proceso de cambio, haciendo que los partidos de derecha conserven el poder de veto.

Ecuador fue a la saga y, en ese mismo mes de octubre de 2019, las protestas en las calles hicieron escuchar su rechazo a la Carta de Intención firmada con el Fondo Monetario Internacional por el gobierno de Lenin Moreno. En este caso, el detonante del conflicto fueron los recortes a los subsidios al combustible y los derechos laborales de los empleados públicos. De forma inmediata, las demandas de estos sectores se vieron acompañadas por colectivos de mujeres, estudiantes y pueblos originarios, estos últimos con una valiosa historia y gran capacidad de organización política en el país.

En definitiva, los estallidos sociales y las luchas populares no se detuvieron durante el embate de las derechas en América Latina. El escenario actual ha renovado las esperanzas de muchas y muchos. Recientemente, el Movimiento Al Socialismo (MAS) triunfó en las elecciones en Bolivia. Luis Arce y David Choquehuanca son ya los nuevos presidente y vicepresidente de ese país. Asimismo, el líder depuesto Evo Morales regresó a su tierra natal en una caravana épica, acompañado de líderes populares y movimientos sociales que son símbolo de las luchas en la región.

A esto se suman los magros resultados electorales del candidato oficialista en las elecciones municipales de São Paulo en Brasil y, en el plano de política internacional, se suma también la derrota de Donald Trump en Estados Unidos. 
En definitiva, el modelo neoliberal de acumulación del capital, las resistencias populares a esos despojos, la construcción de fuerzas políticas en el campo popular y las estrategias de gobierno de las "nuevas" derechas son, en su conjunto, procesos diferentes pero interrelacionados. Este dossier quiere hacer aportes para entenderlos en su complejidad y su carácter polifacético. El volumen consta de 17 artículos. Los primeros cinco presentan un panorama variado acerca de las ideas y prácticas de gobierno en el neoliberalismo latinoamericano. Les siguen otros dos que plantean visiones supranacionales en cuanto a los procesos de resistencia e integración regional; y otros tres que estudian la cuestión de las protestas sociales, tanto desde el punto de vista teórico como a partir de referencias empíricas. La segunda mitad del dossier se compone de cinco artículos referidos al espacio rural, los procesos de alianzas y las prácticas de resistencia allí desplegados. El dossier se cierra con otros dos artículos que abordan dos segmentos críticos de la vida social actual y que son escenario de las disputas más brutales de la actualidad: la producción de energía y la de alimentos. A continuación, pasamos revista detallada de cada uno de los trabajos reseñados.

En "Elementos para una genealogía del movimiento neoliberal en Argentina: Intelectuales, políticos y empresarios", María Paula de Büren reconstruye una necesaria genealogía de las redes neoliberales en ese país y permite recuperar parte de sus despliegues iniciales, durante los años 50 y 60, trazando un hilo de continuidad hasta la actualidad. La autora señala la centralidad que supo tener entonces la figura de Alberto Benegas Lynch y el Centro de Difusión de la Economía Libre (CDEL) fundado por él. Así, de Büren muestra que, lejos de ser resultado de influencias principalmente norteamericanas, la instalación original de las corrientes de pensamiento neoliberal en el país tempranamente tuvo influencias de la vertiente austríaca articulada en torno de la Mont Pèlerin Society.

El trabajo de Gina Paola Rodríguez “Think Tanks de derecha y discursos de género en Chile" es un excelente complemento del primero, en tanto nos abre el camino para comprender la importancia actual de los think tanks, decodificar sus ideas y atender a sus eventuales impactos en el diseño de políticas públicas en la región. Rodríguez analiza los discursos de género actualmente sostenidos por tres think tanks (Li- 
bertad y Desarrollo, Fundación para el Progreso y Avanza Chile). Afirma que la estrategia adoptada por estos actores para librar la "guerra de ideas" contra el progresismo consistió en adaptar de forma parcial y selectiva las banderas del feminismo, ignorando, al mismo tiempo, los derechos de disidencias sexo-genéricas y colectivos LGTBIQ.

A continuación, Diana Villegas Loeza ("Análisis de la relación entre el Estado, ONG y poblaciones rurales en México") aporta elementos para pensar estrategias de gobierno desde una perspectiva etnográfica e histórica. Tras revisar las relaciones de gobierno articuladas durante el Estado de bienestar, la autora estudia la reconfiguración de la relación de este con las poblaciones campesinas e indígenas en el contexto neoliberal, adentrándose entonces en los años 2000. Su trabajo se concentra en el municipio de Pahuatlán, localizado en la Sierra Norte de Puebla, y plantea que la intervención de agencias de desarrollo y las ONG microfinancieras han avanzado con procesos de bancarización en zonas rurales del país. En estos esquemas, plantea, los sujetos ya no son estrictamente beneficiarios ni mucho menos portadores de derechos, sino clientes, socios y/o usuarios que, tras el discurso del "empoderamiento", son presentados como "empresarios de sí mismos".

El trabajo de Emilio Seveso dialoga estrechamente con estas cuestiones. Titulado "Mercantilización en las políticas de inclusión al trabajo: una aproximación desde las experiencias", el texto problematiza los mecanismos de asistencia social condicionada en la provincia de San Luis (Argentina) durante los años recientes. El autor estudia las vivencias y expresiones de beneficiarios del Subprograma Concertación con la Comunidad a la luz de tres dimensiones: la expropiación de la vitalidad corporal vía valorización del capital humano, la circulación monetaria promovida mediante la aplicación de los programas y la desposesión lograda a través de la convergencia de créditos para el consumo. El texto resulta provocativo al sugerir que las energías y los cuerpos de los beneficiarios fueron puestos al servicio de una agenda de "seguridad", al tiempo que guiados por lógicas de endeudamiento directo como vía de incentivo al consumo.

El texto de Emanuel Farías Carrión se titula "Pronunciamiento institucional del neoliberalismo chileno". El mismo procura revisar el reciente Acuerdo Nacional por la Paz y la Nueva Constitución en paralelo a lo que fue el Acuerdo Nacional para la Transición a la Plena Demo- 
cracia, pactado durante la dictadura de Pinochet. Con su propuesta, el autor nos invita a mirar el presente inmediato de la sociedad chilena con una mirada atenta a la historia. A partir de allí, pone en duda la "real pretensión democratizadora" del reciente acuerdo, y señala que este, como aquel, lleva consigo dimensiones que anulan la participación popular y dan continuidad al programa neoliberal en ese país.

En el plano de la integración regional, el texto "El ALBA-TCP: De la emergencia a la resistencia" de María del Carmen Pérez ofrece una pregunta provocativa: ¿está realmente muerto el ALBA? La autora propone pensar la Alianza Bolivariana para los Pueblos de Nuestra América, a partir de sus momentos de crecimiento (2004-2015) y de estancamiento y resistencia (2015 al presente). El objetivo del texto es situar estos hechos en un presente en el cual no se descarte, al menos no mecánicamente, la posibilidad de resurgimiento o potenciación de ese tratado internacional. La actualidad del planteo queda demostrada en la reciente asunción de Arce a la presidencia boliviana, pues reflotar el ALBA es una de las banderas levantadas por el MAS.

Atendiendo al escenario global, pero enfocando la mirada en las luchas campesinas, Carolina Cepeda Másmela y Julián Muñoz Londoño presentan un estudio respecto de Colombia, referido a las relaciones que el Coordinador Nacional Agrario y la Federación Nacional Sindical Unitaria Agropecuaria (Fensuagro) mantienen con La Vía Campesina. Los autores estudian las líneas programáticas vigentes de ambos colectivos, así como también reseñan las articulaciones actualmente mantenidas por ellos. El texto aporta elementos para entender los procesos de articulación global de los movimientos campesinos.

El artículo de María Virginia Quiroga y Ana Lucía Magrini se titula "Protestas sociales y cuestión social en América Latina contemporánea" e indaga acerca de los usos recientes de la noción de protesta social. Las autoras propone pensar este fenómeno "en plural", a partir de su heterogeneidad, y como un objeto de estudio que no termina de calzar por completo en una disciplina o campo de saber delimitado. Su enfoque multidimensional la lleva a proponer cuatro dimensiones constituyentes de "las protestas": políticas, comunicativas, históricas y socio-territoriales, independientemente de la posición que las mismas ocupen en el espectro político. El trabajo de Quiroga y Magrini cierra con una reflexión de las limitaciones que el escenario de pandemia 
está imponiendo a los movimientos sociales, así como los cambios que pueden observarse en los repertorios de acción de la resistencias populares en ese mismo contexto.

También Iván Mézquita Alonso, Laura Celina Ruelas Monjardín y Noé Hernández Cortez proponen avanzar en una caracterización de las dinámicas contenciosas. En este caso, lo hacen a propósito de los conflictos socioambientales producidos en torno de la construcción de hidroeléctricas en México (estados de Nayarit, Guerrero, Sinaloa, Puebla, Jalisco, Veracruz, Oaxaca e Hidalgo) y Colombia (departamentos de Huila y Caldas) durante los últimos tres lustros. El texto, denominado "Hacia una tipología de conflictos socioambientales en hidroeléctricas: experiencias de casos mexicanos y colombianos", analiza los niveles de participación ciudadana en la toma de decisiones según los niveles de conflicto, encontrando que los mayores grados de este tipo de participación en el proceso de construcción de las represas reducen las probabilidades de conflictividad social y la intensidad de los eventuales conflictos.

El artículo "Movilización cívica, redes sociales y neoliberalismo: resistencias en México y Ecuador, 2018-2019", de Daniel Javier de la Garza Montemayor y Gabriela Estefanía Riera Robles, analiza las dinámicas de movilización cívica y las nuevas formas de organización a través de las redes sociales. A partir de allí, los autores procuran detectar la relevancia que ha tenido la articulación vía internet en la campaña electoral de Andrés Manuel López Obrador, en México, y en la generación de las movilizaciones ecuatorianas de octubre de 2019. De la Garza y Riera Robles interpretan que ambos fueron momentos cuando la esfera pública virtual se convirtió en un espacio de articulación y de oposición al liberalismo.

Los artículos de María Amalia Miano, Daniela Pessolano, Carla Sabrina Aguirre y Mariana Ortega atienden distintas experiencias de organización en el espacio rural argentino y mexicano. El trabajo de Miano, denominado "Vivir, educar y luchar en el campo. Acciones y coaliciones de pobladores rurales", analiza dos casos de organización y vida rural comunitaria de la actualidad: familias que participan del Consejo de Administración de un Centro Educativo para la Producción Total (provincia de Buenos Aires) y una Escuela de la Familia Agrícola (EFA) al noreste de la provincia de Chaco. Apoyándose en investigacio- 
nes de tipo colaborativas, el artículo observa que hay acciones de los pobladores rurales que, aún pareciendo minúsculas, merecen ser entendidas como acciones políticas, en tanto permiten a los pobladores rurales sostener las formas de vida que ellos eligen.

Daniela Pessolano, por su parte, se pregunta respecto de los aportes que las mujeres están haciendo a la conformación del espacio social campesino en la provincia de Mendoza (Argentina). En su título el artículo recupera un interrogante punzante: “¿Las mujeres son más campesinas?". Basado en un sólido trabajo de campo, el texto muestra el lugar central que las mujeres campesinas actualmente tienen en la reproducción cotidiana de la vida familiar y la responsabilidad que detentan a la hora de la construcción de procesos asociativos.

En "Extractivismo, territorio y autonomía indígena. El pueblo mapuche en Neuquén (1996-2015)" Carla Sabrina Aguirre se pregunta por las alianzas que la Confederación Mapuche de Neuquén, a lo largo de dos décadas, ha conseguido construir por fuera del espacio indígena. La autora observa que dichas estrategias resultan provechosas para la consecución de objetivos políticos de la organización. Lo hace apoyándose en experiencias de trabajo de campo y concentrando la mirada en dos comunidades (Paichil Antriao y Puel Pvjv) con características históricas y geográficas marcadamente distintas, aunque ambas agrupadas en la Confederación.

El texto de Mariana Ortega ("Territorios que hablan. Comunicación y resistencias en el norte de Argentina") se centra en los procesos de resistencia indígena en la zona periurbana de Tartagal (provincia de Jujuy) en la actualidad. Analiza, para ello, un proceso organizativo iniciado hace más de dos décadas, gestado por gente wichi, guaraní, qom y chorote, y que dio por resultado la Organización de Mujeres Indígenas Asociación Regional de Trabajadores en Desarrollo (ARETEDE) y la Radio Comunitaria La Voz Indígena (inaugurada en 2008). Ortega nos invita a reconocer en estas experiencias, estrategias de resistencias en donde confluyen luchas territoriales, ambientales y de género en un contexto de fuerte avance del capitalismo extractivista y de extendida pobreza.

“Temacapulín: amenaza de despojo y resistencia ante la construcción de una gran represa" propone un abordaje desde la psicología so- 
cial sobre las formas de vivir y atravesar el trauma generado desde 2007 en aquella comunidad mexicana (Estado de Jalisco), ante la amenaza de desalojo por la construcción de una represa hidroeléctrica. Su autora, Susana Elvia, plantea que la resistencia colectiva puede mitigar el trauma psicosocial en cuestión; en tanto se trata de una forma de restablecer la confianza, recuperar la cohesión social y el tejido sociocomunitario, y resarcir el daño psicoemocional.

El dossier se cierra con los trabajos de Domingo Rafael Castañeda Olvera y Luis Blacha. El primero estudia los "Impactos ecosociales de los parques eólicos en el Istmo de Tehuantepec, México". Aunque la energía eólica es una energía más "limpia" que otras como el petróleo o el carbón, la instalación de parques eólicos posee consecuencias que no conviene perder de vista. En este texto Castañeda Olvera hace un minucioso recuento del impacto técnico que tienen los proyectos eólicos -implementados en la región desde 1997 en un territorio con amplia mayoría (80\%) de población indígena- y denuncia la falta de consulta a las comunidades y el engaño a los pobladores; el incumplimiento de las expectativas laborales, económicas y energéticas, etc.; la privatización de los recursos energéticos y el despojo biocultural, entre otras cuestiones.

Finalmente, el texto de Luis Blacha se denomina "Riesgo, desigualdad y sabor. Herramientas sociológicas para explicar el 'efecto dorito”. Su título recupera el nombre comercial de un producto ultraprocesado del mercado de alimentos para invitarnos a reflexionar respecto de las desigualdades económicas y sociales que actualmente se plasman en las capacidades diferenciales para acceder al derecho a la alimentación nutritiva y saludable. El autor resitúa la vieja pregunta sociológica relacionada con el gusto en un esquema actualizado y abarcativo de reflexión que conjuga el escenario de monocultivos, la realidad de la industria alimenticia global, la "falsa sensación de abundancia" plasmada en las góndolas de los supermercados y el consumo popular de productos "doritos": cada vez con menos nutrientes, pero más sabrosos, para garantizarse la "captura" del consumidor.

\section{Referencias}

Giordano, V. (2011). Alegato a favor de una sociología histórica comparada para América Latina. Trabajo y Sociedad, 
15(17), 41-48. Recuperado de https://www.redalyc.org/ pdf/3873/387334689003.pdf

Giordano, V. (2014).¿Qué hay de nuevo en las "nuevas derechas"? Nueva Sociedad, 254. Recuperado de https://nuso.org/articulo/ que-hay-de-nuevo-en-las-nuevas-derechas/

Giordano, V. y Rodríguez, G. (en prensa). Las mujeres de las derechas latinoamericanas en el siglo XXI. CIDOB d'Afers Internacionals.

Leone, M. (2018). Seguritización de la indigeneidad. La actual política indigenista de Argentina y Chile. Question, 1(59), 1-25. https://doi.org/10.24215/16696581e075

Leone, M. (en prensa). Racionalidades seguritarias sobre el pueblo mapuche. Un análisis comparativo de las políticas estatales en Chile y Argentina. Revista Temas y Debates.

Tilly, C. (1991). Grandes estructuras, procesos amplios, comparaciones enormes. Madrid: Alianza Editorial. 